B. I. Freedman - F. C. Hsu - C. D. Langefeld •

S. S. Rich · D. M. Herrington · J. J. Carr · J. Xu •

D. W. Bowden $\cdot$ L. E. Wagenknecht

\title{
The impact of ethnicity and sex on subclinical cardiovascular disease: the Diabetes Heart Study
}

Received: 22 June 2005 / Accepted: 4 August 2005 / Published online: 1 November 2005

(C) Springer-Verlag 2005

\begin{abstract}
Aims/hypothesis: African Americans with type 2 diabetes and access to adequate healthcare are at lower risk of clinical coronary artery disease than are white diabetic patients. We evaluated whether ethnic differences in subclinical cardiovascular disease, coronary and carotid artery calcified plaque and carotid artery intima-medial thickness (IMT) were present in members of The Diabetes Heart Study families. Subjects and Methods: In a biracial cohort of 1,180 individuals from families enriched for members with type 2 diabetes, we calculated coronary and carotid artery calcified plaque using fast-gated helical computed tomography, and measured carotid artery IMT and clinical risk factor profiles. Generalised estimating equations were used to test for an association between measures of subclinical cardiovascular disease and ethnicity and sex. Results: After adjustment for age, ethnicity and kidney function, African Americans had significantly lower amounts of coronary artery calcified plaque (mean \pm SE) $(866 \pm 158$
\end{abstract}

B.I. Freedman and F.C. Hsu contributed equally to the preparation of this manuscript

B. I. Freedman $(\bowtie) \cdot$ D. M. Herrington · D. W. Bowden

Wake Forest University School of Medicine,

Department of Internal Medicine/Section on Nephrology,

Medical Center Boulevard,

Winston-Salem, 27157-1053, NC, USA

e-mail: bfreedma@wfubmc.edu

Tel.: +1-336-7166192

Fax: $+1-336-7164318$

F. C. Hsu $\cdot$ C. D. Langefeld · S. S. Rich ·

J. Xu · L. E. Wagenknecht

Public Health Sciences,

Wake Forest University School of Medicine,

Winston-Salem, NC, USA

\section{J. J. Carr}

Radiologic Sciences,

Wake Forest University School of Medicine,

Winston-Salem, NC, USA

D. W. Bowden

Biochemistry/Center for Human Genomics,

Wake Forest University School of Medicine,

Winston-Salem, NC, USA vs $1,915 \pm 135$, respectively; $p=0.0466)$ and carotid artery calcified plaque ( $179 \pm 51$ vs $355 \pm 27$, respectively; $p=0.0240$ ) relative to whites, despite having increased carotid IMT $(0.71 \pm 0.01$ vs $0.67 \pm 0.004 \mathrm{~cm}$, respectively; $p=0.0007)$, and higher blood pressure, albuminuria and $\mathrm{HbA}_{1} \mathrm{c}$. Sex-specific analyses revealed that African American men had significantly lower coronary and carotid artery calcified atheroma than white men. In women, ethnic differences in calcified carotid artery plaque, but not coronary artery plaque, were observed. Conclusions/interpretation: In families enriched for members with type 2 diabetes, African American men had markedly lower levels of coronary and carotid artery calcified plaque than white men, despite increased carotid artery IMT and conventional risk factors. These findings suggest that susceptibility to subclinical cardiovascular disease differs markedly according to ethnicity and sex.

Keywords African American - Carotid intima-medial thickness · Coronary artery calcium - Ethnicity $\cdot$ Gender . Sex Type 2 diabetes mellitus

Abbreviations ACR: albumin : creatinine ratio . CABG: coronary artery bypass graft $\cdot \mathrm{CCA}$ : common carotid artery - CT: computed tomography . CVD: cardiovascular disease - DHS: Diabetes Heart Study - GEE: generalised estimating equation · IMT: intima-medial thickness - MESA: Multi-Ethnic Study of Atherosclerosis - NHANES: National Health and Nutrition Examination Survey · VA: Veterans' Administration - WFUSM: Wake Forest University School of Medicine

\section{Introduction}

Diabetes mellitus is an independent risk factor for the development of atherosclerotic cardiovascular disease (CVD) and nephropathy [1-5]. In the overall population, diabetic African Americans disproportionately suffer from myocardial infarction, stroke and end-stage renal disease relative to 
white diabetic patients [6-8]. Ethnic differences in access to preventive healthcare, medications, coronary artery bypass surgery and catheter-based coronary interventions clearly contribute to this disparity [9-14], although recent reports suggest that control of diabetes and related risk factors is suboptimal in many African American, white and Hispanic-American diabetics $[15,16]$.

Paradoxically, when insurance status and access to healthcare are equivalent, African Americans have significantly lower rates of clinical coronary artery disease than whites. Karter et al. [17] evaluated 62, 432 diabetic individuals insured by Kaiser Permanente to assess whether access to healthcare was associated with ethnic disparities in diabetes complications. Although there were no statistically significant differences between African Americans relative to whites in overall risk of stroke, congestive heart failure or lower extremity amputation, the adjusted African American:white hazard ratio was 0.56 for myocardial infarction. Young et al. [18] performed a longitudinal cohort study in 429,918 diabetic veterans cared for in a national healthcare system (Veterans' Administration [VA]). A $27-49 \%$ lower rate of CVD was observed in ethnic minorities vs whites. A marked survival advantage is also observed among African Americans receiving renal replacement therapy, an effect that is independent from the delivered dose of dialysis [19-23]. Diabetic subjects having renal replacement therapy have access to Medicare insurance coverage; thus the effect of prior healthcare disparities may be minimised. These data suggest that African Americans may be less susceptible than whites to hyperglycaemia-induced macrovascular disease.

The prevalence and incidence rate of CHD increases with increasing coronary calcium score [24, 25]. Despite markedly higher rates of CHD risk factors among African Americans relative to whites, there is a growing body of evidence that hypertensive African Americans have lower levels of coronary artery calcified plaque [26-31]. Prior reports have not evaluated interactions between ethnicity and calcified vascular plaque among type 2 diabetic subjects with preserved renal function. This study was performed to detect and quantify ethnic differences in calcified atheromatous plaque in the coronary and carotid arteries of subjects with type 2 diabetes mellitus enrolled in The Diabetes Heart Study (DHS) [32-34].

\section{Subjects and methods}

Study population The DHS evaluated a bi-racial cohort of families, each containing at least two siblings concordant for type 2 diabetes. When possible, one non-diabetic sibling from each family was recruited. Families were identified through community advertising or subjects treated in local general internal medicine and endocrinology clinics in northwestern North Carolina. Entry criteria have been reported previously [32-34]. In brief, index cases having diabetes diagnosed after the age of 34 years (in the absence of historical evidence of diabetic ketoacidosis) and at least one additional type 2 diabetic sibling were recruited. The study was approved by the Institutional Review Board at the Wake Forest University School of Medicine (WFUSM) and all participants gave written informed consent.

Examinations were conducted in the General Clinical Research Center of the WFUSM and included interviews for medical history, current medications and health behaviours, measurements of body size, resting blood pressure, 12-lead electrocardiogram, fasting blood draw and spot urine collection. Laboratory assays included urinary albumin and creatinine (to calculate an albumin : creatinine ratio $[\mathrm{ACR}]$ ), total cholesterol, LDL, HDL, triglycerides, $\mathrm{HbA}_{1} \mathrm{c}$, highly sensitive $\mathrm{C}$-reactive protein, fasting serum glucose, blood urea nitrogen, serum creatinine and albumin concentrations. GFR were computed according to Modification of Diet in Renal Disease recommendations [35]. History of CVD was provided by participant self-report. Hypertension was considered present if the participant was previously diagnosed, took anti-hypertensive medications, or had a blood pressure $>140 / 90 \mathrm{mmHg}$ at the study visit. We excluded subjects with an elevated serum creatinine concentration $(\geq 141 \mathrm{mmol} / 1$ in men, $\geq 124 \mathrm{mmol} / 1$ in women) and those reporting prior coronary artery bypass surgery or carotid endarterectomy, due to the marked effects these procedures would have on vascular calcium score.

Vascular imaging Estimates of coronary and carotid artery calcified plaque were made using fast-gated helical computed tomography (CT) [34]. Scans were performed in duplicate after 3-min rest periods. Cardiac CT examinations were performed on a single-slice subsecond helical CT or a four-channel multidetector CT both with cardiac gating and capable of $500 \mathrm{~ms}$ temporal resolution (HiSpeed LX and LightSpeed QXi with the SmartScore Cardiac scan package; General Electric Medical Systems, Waukesha, WI, USA). Scan parameters were 3-mm slice thickness, 26-cm display field-of-view, retrospective cardiac gating, $120 \mathrm{kV}, 240 \mathrm{~mA}$ and $\mathrm{CT}$ scan pitch adjusted to heart rate for the single-slice system and $2.5-\mathrm{mm}$ slice thickness in 4-slice mode, 26-cm display field-of-view, prospective cardiac gating at $50 \%$ of the RR interval, $120 \mathrm{kV}, 240 \mathrm{~mA}$ for the multidetector CT.

For the carotid examination an un-enhanced CT scan was performed through the neck after instructing the participant to swallow [32]. The start and end locations were the $\mathrm{C} 2-3$ and $\mathrm{C} 6-7$ disc levels. A helical acquisition using a 3-mm (single-slice) or 2.5-mm (multidetector) slice columniation, a $120 \mathrm{kV}, 280 \mathrm{~mA}, 0.8$-s gantry rotation, $360^{\circ}$ scan reconstruction and standard reconstruction kernel was performed. The display field of view was $18 \mathrm{~cm}$ resulting in pixel dimensions of $0.35 \times 0.35 \mathrm{~mm}$.

CT examinations in both vascular beds were analysed on a GE Advantage Windows Workstation using the research version SmartScores software package (General Electric), which allows calculation of a calcium mass score of the amount of calcified plaque. The reproducibility estimates 
of the coronary and carotid calcified plaque scores obtained from the duplicate scans were both 0.98. Two trained readers blinded to the clinical characteristics of the participants analysed the CT images. As previously reported, helical CT scans yield comparable vascular calcium measurements to electron beam CT [36]. The cardiac CT methods that we used in the DHS replicate those employed in the Multi-Ethnic Study of Atherosclerosis (MESA) and Coronary Artery Risk Development In Young Adults studies [37].

Common carotid artery (CCA) intima-medial thickness (IMT) was measured using B-mode ultrasound, as reported previously [33]. Briefly, high-resolution B-mode carotid ultrasonography was performed using a $7.5-\mathrm{MHz}$ transducer and a Biosound Esaote (AU5) machine. Scans were performed of the near and far walls of the distal $10-\mathrm{mm}$ portion of the CCA at five predefined interrogation angles on each side. The mean value of up to 20 CCA IMT values is reported here.

Statistical methods The sample means, SD and medians were computed for the continuous characteristics and the measures of subclinical CVD (coronary and carotid artery calcified plaque and carotid IMT). For the discrete demo-

Table 1 The impact of ethnicity and sex on CVD risk factors in DHS participants ${ }^{\mathrm{a}}$

\begin{tabular}{|c|c|c|c|c|c|c|}
\hline & \multicolumn{2}{|l|}{ White $(n=1000)$} & \multicolumn{2}{|c|}{ African American $(n=180)$} & \multicolumn{2}{|l|}{$p$-value } \\
\hline & Female $(n=529)$ & Male $(n=471)$ & Female $(n=122)$ & Male $(n=58)$ & Ethnicity $^{\mathrm{b}}$ & $\mathrm{Sex}^{\mathrm{c}}$ \\
\hline Age (years) & $61.4(9.4) 60.8$ & $61.9(9.3) 62.6$ & $58.5(9.4) 57.6$ & $58.9(8.6) 58.7$ & 0.0013 & 0.9031 \\
\hline T2DM duration (years) & $10.1(6.8) 8$ & $10.8(7.5) 9$ & $10.3(7.6) 8$ & $11.3(8.4) 9$ & 0.4259 & 0.4269 \\
\hline T2DM (\%) & 80 & 86 & 88.5 & 91.4 & 0.0178 & 0.0469 \\
\hline \multicolumn{7}{|l|}{ T2DM treatment } \\
\hline Insulin (\%) & 24 & 20 & 39.3 & 32.8 & 0.0011 & 0.1222 \\
\hline Oral hypoglycaemic (\%) & 59 & 69.4 & 59 & 62.1 & 0.6644 & 0.0025 \\
\hline Lipid-lowering medication (\%) & 39 & 47.1 & 35.2 & 31 & 0.1124 & 0.0271 \\
\hline BMI $\left(\mathrm{kg} / \mathrm{m}^{2}\right)$ & $32.6(7.5) 31.1$ & $30.9(5.5) 30.1$ & $35(7.3) 34.5$ & $30.9(6.4) 30.5$ & 0.1275 & $<0.0001$ \\
\hline Systolic blood pressure (mmHg) & 139.8 (19.6) 138 & $137.8(18.6) 137$ & $143.9(21.5) 142.5$ & $140(18.8) 139.8$ & 0.0041 & 0.0182 \\
\hline Diastolic blood pressure (mmHg) & $72.2(9.8) 71.3$ & $73.8(10.8) 73.5$ & $76.2(12.1) 75.5$ & 77.3 (10.4) 78 & 0.0009 & 0.0200 \\
\hline \multicolumn{7}{|l|}{ Laboratory } \\
\hline Total cholesterol (mmol/l) & $5.10(1.11) 5.00$ & $4.63(1.03) 4.56$ & $5.09(0.99) 5.08$ & $4.63(0.88) 4.51$ & 0.6892 & $<0.0001$ \\
\hline HDL (mmol/l) & $1.24(0.35) 1.19$ & $0.99(0.25) 0.96$ & $1.39(0.41) 1.37$ & $1.15(0.31) 1.11$ & 0.0001 & $<0.0001$ \\
\hline LDL (mmol/l) & $2.82(0.84) 2.75$ & $2.65(0.82) 2.59$ & $3.04(0.92) 2.99$ & $2.81(0.79) 2.72$ & 0.0198 & 0.0008 \\
\hline Triglycerides (mmol/1) & 2.38 (1.57) 2.02 & $2.26(1.49) 1.90$ & $1.50(0.89) 1.24$ & $1.54(1.29) 1.13$ & $<0.0001$ & 0.0332 \\
\hline $\mathrm{HbA}_{1} \mathrm{c}$ (proportion) & $0.073(0.019) 0.07$ & $0.073(0.017) 0.071$ & $0.087(0.03) 0.077$ & $0.084(0.021) 0.08$ & $<0.0001$ & 0.7566 \\
\hline Fasting glucose $(\mathrm{mmol} / \mathrm{l})$ & $7.86(3.33) 6.99$ & $7.77(3.06) 7.16$ & $8.39(3.93) 7.55$ & $8.86(5.23) 8.05$ & 0.2302 & 0.7273 \\
\hline Serum creatinine $(\mathrm{mmol} / \mathrm{l})$ & $88.4(26.5) 88.4$ & $106.1(26.5) 106.1$ & $97.2(35.4) 88.4$ & 114.9 (44.2) 114.9 & 0.0015 & 0.5031 \\
\hline Urine ACR (mg/mmol) & $13.8(71.9) 1.3$ & $12.1(42.5) 1.5$ & $29.3(93.7) 1.9$ & $35.2(83.2) 2.1$ & 0.0015 & 0.5031 \\
\hline GFR (ml/s) & $1.10(0.29) 1.05$ & $1.18(0.31) 1.15$ & $1.23(0.40) 1.24$ & $1.26(0.34) 1.29$ & 0.0008 & $<0.0001$ \\
\hline C-reactive protein $(\mu \mathrm{mol} / \mathrm{l})$ & $0.06(0.08) 0.03$ & $0.04(0.08) 0.02$ & $0.06(0.06) 0.04$ & $0.05(0.06) 0.03$ & 0.7028 & $<0.0001$ \\
\hline \multicolumn{7}{|l|}{ Smoking } \\
\hline Current $(\%)$ & 16 & 17.6 & 17.2 & 43.1 & 0.0292 & 0.0141 \\
\hline Past $(\%)$ & 56.3 & 23.1 & 50.8 & 10.3 & 0.7436 & $<0.0001$ \\
\hline Never $(\%)$ & 27.2 & 59 & 30.3 & 46.6 & & \\
\hline Pack-years (current) & $29.2(25.8) 25$ & $40.1(30.4) 36$ & 16.5 (17) 10.5 & $25.6(25.5) 21$ & 0.0004 & $<0.0001$ \\
\hline \multicolumn{7}{|c|}{ Prevalent cardiovascular conditions, procedures } \\
\hline Hypertension $(\%)^{\mathrm{d}}$ & 68 & 62.2 & 73.8 & 65.5 & 0.2149 & 0.0006 \\
\hline Angina (\%) & 14 & 20.1 & 17.2 & 8.6 & 0.6752 & 0.0697 \\
\hline Stroke $(\%)$ & 7.9 & 11.3 & 5.7 & 6.9 & 0.3540 & 0.1153 \\
\hline Heart attack (\%) & 10.6 & 29.5 & 9 & 6.9 & 0.0196 & $<0.0001$ \\
\hline CABG (\%) & 5.5 & 24 & 5.7 & 5.2 & 0.0409 & $<0.0001$ \\
\hline Coronary angioplasty (\%) & 10.4 & 20.4 & 9.8 & 1.7 & 0.0228 & $<0.0001$ \\
\hline Carotid endarterectomy (\%) & 1.3 & 3.4 & 0.8 & 0 & 0.2630 & 0.0666 \\
\hline
\end{tabular}

${ }^{a}$ Variables reported as mean $(\mathrm{SD})$ median

${ }^{\mathrm{b}} p$ values for ethnicity are from the GEE model, after adjusting for age and sex

c $p$ values for sex are from the GEE model, after adjusting for ethnicity and age

${ }^{\mathrm{d}}$ Hypertension as defined in the text

$T 2 D M$ type 2 diabetes mellitus 
Table 2 The impact of ethnicity, sex and GFR on subclinical CVD in DHS participants ${ }^{\mathrm{a}}$

\begin{tabular}{|c|c|c|c|c|c|c|c|}
\hline & \multicolumn{2}{|l|}{ White $(n=1000)$} & \multicolumn{2}{|c|}{ African American $(n=180)$} & \multicolumn{3}{|l|}{$p$-value } \\
\hline & Female $(n=529)$ & Male $(n=471)$ & Female $(n=122)$ & Male $(n=58)$ & Ethnicity $^{\mathrm{b}}$ & $\operatorname{Sex}^{\mathrm{c}}$ & $\mathrm{GFR}^{\mathrm{d}}$ \\
\hline Coronary artery calcium & 828 (1151) 110 & $2988(4355) 1336$ & $687(1502) 48$ & $1233(2583) 166$ & 0.0466 & $<0.0001$ & 0.0205 \\
\hline Carotid artery calcium & $244(609) 25$ & 465 (763) 36 & $186(624) 10.5$ & $195(585) 14.5$ & 0.0007 & $<0.0001$ & 0.0747 \\
\hline Carotid IMT (mm) & $0.64(0.10) 0.62$ & $0.71(0.14) 0.68$ & $0.66(0.11) 0.64$ & $0.74(0.13) 0.72$ & 0.0240 & $<0.0001$ & 0.0169 \\
\hline
\end{tabular}

${ }^{a}$ Variables reported as mean (SD) median

${ }^{\mathrm{b}} p$ values for ethnicity are from the GEE model, after adjusting for age, sex and GFR

${ }^{c} p$ values for sex are from the GEE model, after adjusting for age, ethnicity and GFR

${ }^{d} p$ values for GFR are from the GEE model, after adjusting for age, sex and ethnicity

graphic characteristics, the proportions were calculated. A series of generalised estimating equations (GEE) assuming exchangeable correlation and using the empirical estimate of the variance to adjust for familial correlation was computed to test for the relationships between ethnicity, sex and measures of subclinical CVD [38]. To better approximate the distributional assumptions of conditional normality and homogeneity of variance, the natural $\log (\ln )$ of (coronary calcium score +1 ) and $\ln$ (carotid calcium score +1$)$ were analysed. Standard regression diagnostics for collinearity and influence were computed for each model. All statistical analyses were considered significant at $p<0.05$.

\section{Results}

Demographic characteristics of the DHS study population are listed in Table 1. There were 1,000 white participants from 369 families and 180 African American participants from 74 families included in this report. The DHS recruited predominantly white families in order to have adequate statistical power to detect genes underlying susceptibility to subclinical CVD. Among white subjects, 828 were diabetic (423 female and 405 male) and among African American subjects, 161 were diabetic (108 female and 53 male). The African American subjects averaged approximately 3 years younger than the white subjects in this sample. Although younger, a greater percentage of African American participants were taking insulin injections and had diabetes (reflecting the increased recruitment of a non-diabetic sibling in white families). Relative to whites, African American subjects had higher systolic and diastolic blood pressures, $\mathrm{HbA}_{1} \mathrm{c}$, LDL-cholesterol, albuminuria, per cent of current smokers and GFR. Whites had a higher selfreported rate of myocardial infarctions, angina and coronary artery bypass graft (CABG). Sex-related differences were also observed in many clinical and laboratory parameters (Table 1).

Despite the unfavourable risk factor profiles in African Americans and their significantly increased carotid artery IMT, calcified plaque in the coronary and carotid arteries was markedly lower than in whites (Table 2). Marked sex differences in the presence of subclinical CVD were also observed. After adjusting for age, sex and GFR, African American participants had significantly lower coronary artery and carotid artery calcified plaque and increased carotid artery IMT, compared with white subjects. After adjusting for age, sex and ethnicity, GFR was inversely associated with coronary artery calcified plaque and carotid artery IMT, with a trend towards an inverse association with carotid artery calcified plaque (Table 2).

GEE analyses were performed to test whether Ethnicity $\times$ Gender interactions were present in subclinical CVD among the 868 DHS participants who had coronary artery CT scans, 859 with carotid CT scans and 785 with carotid artery IMT who denied prior coronary artery bypass surgery or carotid endarterectomy. Table 3 reveals that despite the absence of Ethnicity $\times$ Gender interactions in carotid IMT, there were significant Ethnicity $\times$ Gender interactions in $\ln$ (coronary artery calcified plaque) and $\ln$ (carotid artery calcified plaque). Thus, the ethnicity effects for coronary artery and carotid artery calcified plaque were different in men and women. We further studied the sex-specific ethnicity effects in Table 4.

Table 4 reveals sex-specific ethnicity differences in measures of subclinical CVD. Lower coronary artery and carotid artery calcified plaque burden was observed in African American men. In African American women a trend towards reduction in carotid artery calcified plaque was observed in the fully adjusted model $(p=0.06)$. In contrast, African American women had significantly increased carotid IMT relative to white women. Similar differences in carotid IMT were observed between African

Table 3 Generalised estimating equations for Ethnicity $\times$ Sex interaction $^{\mathrm{a}}$

\begin{tabular}{ll}
\hline Variable $^{\mathrm{b}}$ & $\begin{array}{l}\text { Ethnicity } \times \text { Sex interaction term } \\
p \text { value }\end{array}$ \\
\hline ln (coronary artery calcium) & $<0.0001$ \\
$\ln$ (carotid artery calcium) & 0.0186 \\
Carotid IMT & 0.8509 \\
\hline
\end{tabular}

${ }^{a}$ Analyses include 712 white and 156 African American participants who had not had prior carotid endarterectomy or coronary artery bypass surgery, with no more than ten values missing for any given analysis/ethnicity subset

${ }^{\mathrm{b}}$ With adjustment for age, sex, ethnicity and GFR 
Table 4 Sex specific ethnicity effects on subclinical CVD

\begin{tabular}{|c|c|c|c|}
\hline \multirow[t]{2}{*}{$\begin{array}{l}\text { Subclinical CVD } \\
\text { measure }\end{array}$} & \multirow[t]{2}{*}{ Covariates in the sex-specific model } & \multicolumn{2}{|c|}{$\begin{array}{l}\text { Estimate (SE) and } p \text { value for African } \\
\text { Americans vs white }\end{array}$} \\
\hline & & Male & Female \\
\hline \multirow[t]{3}{*}{$\begin{array}{l}\ln (\text { coronary artery } \\
\text { calcium) }\end{array}$} & Age, ethnicity, GFR & $\begin{array}{l}-1.23(0.32) \\
p=0.0001\end{array}$ & $\begin{array}{l}-0.02(0.26) \\
p=0.9497\end{array}$ \\
\hline & Age, ethnicity, BMI, hypertension, smoking, ACR, diabetes, CVD, GFR & $\begin{array}{l}-0.95(0.33) \\
p=0.0037\end{array}$ & $\begin{array}{l}-0.26(0.26) \\
p=0.3146\end{array}$ \\
\hline & $\begin{array}{l}\text { Age, ethnicity, BMI, hypertension, smoking, ACR, diabetes, CVD, GFR, } \\
\text { triglyceride, HDL, LDL }\end{array}$ & $\begin{array}{l}-1.02(0.34) \\
p=0.0029\end{array}$ & $\begin{array}{l}-0.22(0.27) \\
p=0.4017\end{array}$ \\
\hline \multirow[t]{3}{*}{$\begin{array}{l}\ln (\text { carotid artery } \\
\text { calcium) }\end{array}$} & Age, ethnicity, GFR & $\begin{array}{l}-0.99(0.40) \\
p=0.0136\end{array}$ & $\begin{array}{l}-0.14(0.20) \\
p=0.4923\end{array}$ \\
\hline & Age, ethnicity, BMI, hypertension, smoking, ACR, diabetes, CVD, GFR & $\begin{array}{l}-1.02(0.40) \\
p=0.0119\end{array}$ & $\begin{array}{l}-0.41(0.21) \\
p=0.0482\end{array}$ \\
\hline & $\begin{array}{l}\text { Age, ethnicity, BMI, hypertension, smoking, ACR, diabetes, CVD, GFR, } \\
\text { triglyceride, HDL, LDL }\end{array}$ & $\begin{array}{l}-0.97(0.42) \\
p=0.0203\end{array}$ & $\begin{array}{l}-0.42(0.22) \\
p=0.0613\end{array}$ \\
\hline \multirow[t]{3}{*}{ Carotid IMT (mm) } & Age, ethnicity, GFR & $\begin{array}{l}0.03(0.02) \\
p=0.0909\end{array}$ & $\begin{array}{l}0.04(0.01) \\
p=0.0035\end{array}$ \\
\hline & Age, ethnicity, BMI, hypertension, smoking, ACR, diabetes, CVD, GFR & $\begin{array}{l}0.03(0.02) \\
p=0.1809\end{array}$ & $\begin{array}{c}0.03(0.01) \\
p=0.0111\end{array}$ \\
\hline & $\begin{array}{l}\text { Age, ethnicity, BMI, hypertension, smoking, ACR, diabetes, CVD, GFR, } \\
\text { triglyceride, HDL, LDL }\end{array}$ & $\begin{array}{l}0.04(0.02) \\
p=0.0507\end{array}$ & $\begin{array}{l}0.04(0.01) \\
p=0.0019\end{array}$ \\
\hline
\end{tabular}

${ }^{a}$ Sample sizes are 340 males and 528 females who denied prior carotid endarterectomy or coronary artery bypass surgery, with no more than 28 male or 32 female participants missing in any model

American and white men; however, the $p$-value attained statistical significance only in the fully adjusted model.

\section{Discussion}

This report is the first to examine a large bi-racial cohort of individuals with type 2 diabetes for the presence of ethnic and sex-related differences in subclinical CVD. We identified significantly lower calcified atheromatous plaque burden in the coronary and carotid arteries of diabetic African Americans than whites. Sex-specific effects analysis revealed that African American men had significantly lower coronary artery and carotid artery calcified atheroma than white men, and African American women had lower carotid artery calcified atheroma than white women after adjustment for age, BMI, hypertension, smoking, ACR, presence of diabetes, GFR and CVD. Racial differences in calcified coronary artery plaque were not observed in women. These findings, observed despite poorer glycaemic control, generally adverse CVD risk factor profiles and increased carotid artery IMT in African Americans, support the hypothesis that the pathological significance of calcified atherosclerotic plaque varies according to ethnicities and sex. Our results replicate those observed in prior reports measuring calcified atherosclerotic plaque using fluoroscopy and electron-beam CT in predominantly nondiabetic cohorts [26-31]. The MESA results in 2,619 white participants (7.8\% were diabetic) and 1,898 African American participants $(20.6 \%$ were diabetic) revealed that $70.4 \%$ of white men and $52.1 \%$ of African American men had detectable coronary calcium $(p<0.001)$, with median calcium scores of 48 in white men and 3 in African American men $(p<0.001)$. We observed higher calcium scores than MESA since the DHS evaluated a population with long-standing and poorly controlled diabetes mellitus.

A recent prospective study demonstrated that calcified coronary artery plaque adds significantly to the prediction of myocardial infarction and CVD death, even in individuals classified as 'low risk' by the Framingham Risk Index/National Cholesterol Education Program Adult Treatment Panel III, the standard metric of traditional CVD risk factors [39]. Although vulnerable plaque is not clearly defined in the American Heart Association histological classification of advanced atherosclerotic lesions [40], it reflects a potentially thin-walled fibrous cap overlying a lipid core. DHS CT scans measure the calcified component of coronary plaque (American Heart Association $\mathrm{Vb}$ type). However, recent intra-coronary ultrasound data indicate that 'spotty calcifications' are indicative of the culprit lesion in individuals with acute myocardial infarction or unstable angina [41]. Biomechanical studies of the vessel wall indicate that the shoulders of calcified plaques may in fact be the 'vulnerable' regions. Thus, calcified plaque may play an important role in predicting acute CVD events.

It is estimated that $14.9 \%$ of African Americans have either diagnosed or undiagnosed diabetes mellitus, and an additional 6.3\% have IGT [42]. On average, an African American individual is twice as likely to have diabetes as is his/her white peer [43] and the prevalence of diabetes among African Americans aged 40-74 years has doubled 
from $8.9 \%$ in $1976-1989$ to $18.2 \%$ in $1988-1994$ [44]. Diabetic African Americans have markedly higher overall rates of renal failure and CVD-related mortality compared with white diabetics $[7,8,45]$. Lower socioeconomic status and poorer access to healthcare are presumed to contribute to the adverse outcomes in minority populations [7]. However, reports based on the third National Health and Nutrition Examination Survey I (NHANES) and the Insulin Resistance Atherosclerosis Study suggest that the magnitude of racial differences in healthcare access and utilisation, health status and outcomes for white, African American and Hispanic-American type 2 diabetics has minimal impact on overall health status $[15,16]$. Suboptimal health status relative to established treatment goals (i.e. eye examinations, measurement and treatment of high blood pressure, proteinuria, dyslipidaemia, coronary artery disease, and diabetes treatment with insulin/oral agents) were detected in all racial groups.

Recent analyses in nearly 500,000 type 2 diabetic individuals cared for in health maintenance organisations or the VA suggest that African Americans are actually at markedly lower risk of developing diabetes-related coronary artery disease than whites $[17,18]$. In addition, the NHANES I Epidemiologic Follow-Up Study and the New York City Medical Examiner's Office autopsy study also observed reduced rates of coronary artery disease in African American men, relative to white [46, 47]. These surprising observations reproduce the long-standing and consistent observation that African American dialysis patients survive longer than their white counterparts [19-23]. Lower rates of coronary artery disease-associated mortality, possibly associated with lesser degrees of calcified coronary artery plaque in type 2 diabetic African Americans with access to Medicare-supported renal replacement therapy, may contribute to this survival advantage [22].

We previously addressed ethnic-specific relationships between subclinical atherosclerosis assessed by carotid artery IMT compared with coronary artery and carotid artery calcified plaque in the DHS [32]. Carotid artery IMT is known to predict incident CHD and is recognised by the Food and Drug Administration as a surrogate marker of atherosclerosis progression or regression [48, 49]. Simple and adjusted correlation coefficients between calcium scores and IMT were of moderate size and statistically significant among whites [32]. In contrast, the correlation coefficients were lower and non-significant for these relationships in African Americans. The most striking racial contrast was for the adjusted correlation coefficient between carotid artery calcium and IMT: $0.25(p<0.0001)$ in whites and $-0.10(p=0.64)$ in African Americans. Interaction terms (IMT $\times$ Ethnicity) were tested in models for coronary artery calcium $(p=0.20)$ and carotid artery calcium $(p=0.004)$. In both cases, there was a suggestion (weaker for coronary calcium) that the relationship between IMT and vascular calcium differed between racial groups. A significant, positive relationship between coronary and carotid artery calcium and IMT was observed in whites, not in African Americans.
The inherited and environmental factors that underlie the reduced coronary artery and carotid artery calcified plaque in African Americans, despite similar degrees of carotid artery IMT, remain largely unexplored. The DHS detected strong associations between albuminuria and coronary/ carotid artery calcified atheroma in white diabetic subjects without advanced nephropathy [50]. Albuminuria, more often observed in diabetic African Americans, would be expected to increase calcified plaque in diabetic African Americans, the opposite of what is observed. African Americans ingest less dietary calcium than do whites [51] and oral calcium supplementation lowers blood pressure in African Americans [52]. Reduced dietary calcium intake could conceivably contribute to the observed racial differences in calcified atherosclerotic plaque. However, reduced dietary calcium intake in African Americans is unlikely to fully explain the racial variation, since increased bone mass and reduced rates of osteoporosis are observed in African Americans [51, 53]. African Americans also manifest skeletal resistance to the effects of parathyroid hormone $[51,53]$. It appears likely that racial differences in calcium metabolism contribute to the observed differences in calcified atheroma and bone mineralisation. The deposition of calcium in the arterial wall plaque appears to occur in a process similar to bone formation [54]. The results of the few epidemiological studies that have examined determinants of calcium/bone metabolism and their possible contribution to the variance in coronary artery calcified plaque have been inconsistent.

Potential limitations in our report included the presence of a significantly higher GFR and HDL-cholesterol and reduced levels of triglycerides in African American participants. These effects could translate into protection from the development of calcified atheromatous plaque, since elevated serum triglycerides, reduced renal function and lower HDL-cholesterol levels are potent risk factors for systemic atherosclerosis. We therefore controlled for the variation in GFR and lipid profiles between races and the ethnic disparity in calcified coronary artery plaque persisted. Additionally, differential participation rates between races could have impacted the results. The consistently lower levels of calcified vascular plaque that have been observed in other reports in non-diabetic African Americans make this bias unlikely to fully explain the results.

In summary, reduced amounts of calcified atheromatous plaque were present in the coronary and carotid arteries of African American type 2 diabetic men compared with white. Lower levels of calcified carotid artery plaque were also present in type 2 diabetic African American women, compared with white. These findings were observed despite adverse clinical risk factor profiles in African Americans. Lesser degrees of calcified coronary artery plaque in African Americans are consistent with improved clinical coronary artery disease outcomes among African Americans on dialysis and in those with access to adequate healthcare. Additional studies are urgently needed in order to determine the causes of the ethnic and sex differences that are observed in calcified atheromatous plaque. 
Acknowledgements This study was supported in part by the General Clinical Research Center of the Wake Forest University School of Medicine grant M01 RR07122; NHLBI grant R01 HL67348 (to D. W. Bowden); and by an American Diabetes Association Grant (D. W. Bowden). The investigators acknowledge the cooperation of our participants, the study recruiters Ms Carrie Smith and Ms Sue Ann Backus, the CT analysts Delilah Cook, Susan Pillsbury and Rong Shi, and the CT technologists.

\section{References}

1. Kannel WB, McGee DL (1979) Diabetes and cardiovascular disease. J Am Med Assoc 241:2035-2038

2. Brand F, Abbott RD, Kannel WB (1989) Diabetes, intermittent claudication, and risk of cardiovascular events. The Framingham Study. Diabetes 38:504-509

3. Abbott RD, Donahue RP, MacMahon SW, Reed DM, Yano K (1987) Diabetes and the risk of stroke. J Am Med Assoc 257: 949-952

4. Pan W-H, Cedres LB, Liu K et al (1986) Relationship of clinical diabetes and asymptomatic hyperglycemia to risk of coronary heart disease mortality in men and women. Am J Epidemiol 123:504-516

5. Adler AI, Stevens RJ, Manley SE et al (2003) Development and progression of nephropathy in type 2 diabetes: The United Kingdom Prospective Diabetes Study (UKPDS 64). Kidney Int 63:225-223

6. Clark L, Ferdinand K, Flack JM et al (2001) Coronary heart disease in African Americans. Heart Dis 3:97-108

7. Centers for Disease Control and Prevention, CDC (2004) Disparities in premature deaths from heart disease -50 states and District of Columbia, 2001. MMWR Morb Mortal Wkly Rep 53:121-125

8. US Renal Data System (2003) Annual report: atlas of end-stage renal disease in the United States. National Institutes of Health, National Institutes of Diabetes and Digestive and Kidney Diseases, Bethesda, MD

9. Okelo S, Taylor A, Wright JT Jr, Gordon N, Mohan G, Lesnefsky E (2001) Race and the decision to refer for coronary revascularization: The effect of physician awareness of patient ethnicity. J Am Coll Cardiol 38:698-704

10. Schneider E, Leape L, Weissman JS, Piana R, Gatsonis C, Epstein AM (2001) Racial differences in cardiac revascularization rates: Does 'overuse' explain higher rates among white patients? Ann Intern Med 135:328-337

11. Kressin N, Petersen LA (2001) Racial differences in the use of invasive cardiovascular procedure review of the literature and prescription for future research. Ann Intern Med 135:352-366

12. Ibrahim S, Whittle J, Bean-Mayberry B, Kelley M, Good C, Conigliaro J (2003) Racial/ethnic variations in physician recommendations for cardiac revascularization. Am J Public Health 93:1689-1693

13. Epstein M, Weissman S, Schneider E, Gatsonis C, Leape L, Piana R (2003) Race and gender disparities in rates of cardiac revascularization that reflect appropriate use of procedures or problems in quality care? Med Care 41:1240-1255

14. Chen J, Rathore S, Radford M, Wang Y, Krumholz HM (2001) Racial differences in the use of cardiac catherization after acute myocardial infarction. N Engl J Med 344:1443-1449

15. Harris MI (2001) Racial and ethnic differences in health care access and health outcomes for adults with type 2 diabetes. Diabetes Care 24:454-459

16. Bonds DE, Zaccaro DJ, Karter AJ, Selby JV, Saad M, Goff DC (2003) Ethnic and racial differences in diabetes care: The Insulin Resistance Atherosclerosis Study. Diabetes Care 26:1040-1046

17. Karter AJ, Ferrara A, Liu JY, Moffet HH, Ackerson LM, Selby JV (2002) Ethnic disparities in diabetic complications in an insured population. J Am Med Assoc 287:2519-2527
18. Young BA, Maynard C, Boyko EJ (2003) Racial differences in diabetic nephropathy, cardiovascular disease, and mortality in a national population of veterans. Diabetes Care 26:2392-2399

19. Cowie CC, Port FK, Rust KF, Harris MI (1994) Differences in survival between black and white patients with diabetic endstage renal disease. Diabetes Care 17:681-687

20. Price DA, Owen WF (1997) African Americans on maintenance dialysis: A review of racial differences in incidence, treatment and survival. Adv Ren Replace Ther 4:3-12

21. Owen WF, Chertow GM, Lazarus JM et al (1998) The dose of hemodialysis: Mortality responses by race and gender. J Am Med Assoc 280:1764-1768

22. Bloembergen W, Port FK, Mauger E, Wolfe R (1994) Causes of death in dialysis patients: racial and gender differences. J Am Soc Nephol 5:1231-1242

23. Freedman BI, Soucie JM, Kenderes B et al (2001) Family history of end-stage renal disease does not predict dialytic survival. Am J Kidney Dis 38:547-552

24. Raggi P, Callister TQ, Cooil B et al (2000) Identification of patients at increased risk of first unheralded acute myocardial infarction by electron-beam computed tomography. Circulation 101:850-855

25. Arad Y, Spadaro LA, Goodman K, Newstein D, Guerci AD (2000) Prediction of coronary events with electron beam computed tomography. J Am Coll Cardiol 36:1253-1260

26. Tang W, Detrano R, Brezden OS et al. (1995) Racial differences in coronary calcium prevalence among high-risk adults. Am J Cardiol 75:1088-1091

27. Newman AB, Naydeck BL, Whittle J, Sutton-Tyrrell K, Edmundowicz D, Kuller LH (2002) Racial differences in coronary artery calcification in older adults. Arterioscler Thromb Vasc Biol 22:424-430

28. Khurana C, Rosenbaum CG, Howard BV et al (2003) Coronary artery calcification in black women and white women. Am Heart J 145:724-729

29. Bild DE, Folsom AR, Lowe LP et al (2001) Prevalence and correlates of coronary calcification in black and white young adults: The Coronary Artery Risk Development in Young Adults (CARDIA) Study. Arterioscler Thromb Vasc Biol 21, $852-857$

30. Lee TC, O'Malley PG, Feuerstein I, Taylor AJ (2003) The prevalence and severity of coronary artery calcification on coronary artery computed tomography in black and white subjects. J Am Coll Cardiol 41:39-44

31. Bild DE, Detrano R, Peterson D et al (2005) Ethnic differences in coronary calcification: The Multi-Ethnic Study of Atherosclerosis (MESA). Circulation 111:1313-1320

32. Wagenknecht LE, Langefeld CD, Carr JJ et al (2004) Race specific relationships between coronary and carotid artery calcification and carotid intimal medial thickness. Stroke 35: 97-99

33. Lange LA, Bowden DW, Langefeld CD et al (2002) Heritability of carotid artery intima-medial thickness in type 2 diabetes. Stroke 33:1876-1881

34. Wagenknecht LE, Bowden DW, Carr JJ, Langefeld CD, Freedman BI, Rich SS (2001) Familial aggregation of coronary artery calcium in families with type 2 diabetes. Diabetes 50:861-866

35. Levey AS, Bosch JP, Lewis JB, Greene T, Rogers N, Roth D (1999) A more accurate method to estimate glomerular filtration rate from serum creatinine: A new prediction equation. Ann Intern Med 130:461-470

36. Carr JJ, Crouse JR Jr, Burke GL, D’Agostino RB Jr, Goff DC Jr (2000) Evaluation of subsecond gated helical CT for quantification of coronary artery calcium and comparison with electron beam CT. Am J Roentgenol 174:915-921

37. Carr JJ, Nelson JC, Wong NA et al (2005) Calcified coronary artery plaque measurement with cardiac CT in populationbased studies: Standardized protocol of multi-ethnic study of atherosclerosis (MESA) and coronary artery risk development in young adults (CARDIA) study. Radiology 234:35-43 
38. Liang KY, Zeger SL (1986) Longitudinal data analysis using generalized linear models. Biometrika 73:13-22

39. Greenland P, LaBree L, Azen SP, Doherty TM, Detrano RC (2004) Coronary artery calcium score combined with Framingham score for risk prediction in asymptomatic individuals. J Am Med Assoc 291:210-215

40. Stary HC, Chandler AB, Dinsmore RE et al (1995) A definition of advanced types of atherosclerotic lesions and a histological classification of atherosclerosis. Circulation 92:1355-1374

41. Ehara S, Kobayashi Y, Yoshiyama M et al (2004) Spotty calcification typifies the culprit plaque in patients with acute myocardial infarction. An intravascular ultrasound study. Circulation 110:3424-3429

42. Centers for Disease Control and Prevention, CDC (2005) Health disparities experienced by black or African Americans United States. MMWR Morb Mortal Wkly Rep 54:1-3

43. Brancati FL, Kao WH, Folsom AR, Watson RL, Szklo M (2000) Incident type 2 diabetes mellitus in African American and white adults: The Atherosclerosis Risk in Communities Study. J Am Med Assoc 283:2253-2259

44. Harris MI, Flegal KM, Cowie CC et al (1998) Prevalence of diabetes, impaired fasting glucose, and impaired glucose tolerance in U.S. adults: The Third National Health and Nutrition Examination Survey. Diabetes Care 21:518-524

45. Clark L, Ferdinand K, Flack JM et al (2001) Coronary heart disease in African Americans. Heart Dis 3:97-108

46. Gillum R, Mussolino M, Madans JH (1997) Coronary heart disease incidence and survival in African American women and men. Ann Intern Med 127:111-118
47. Onwuanyi A, Hodges D, Avancha A et al (1998) Hypertensive vascular disease as a cause of death in blacks versus whites. Hypertension 31:1070-1076

48. Chambless LE, Heiss G, Folsom AR et al (1997) An Association of coronary heart disease incidence with carotid arterial wall thickness and major risk factors: The Atherosclerosis Risk in Communities (ARIC) Study, 1987-1993. Am J Epidemiol 146:483-494

49. O'Leary DH, Polak JF, Kronmal RA, Manolio TA, Burke GL, Wolfson SK (1999) Carotid-artery intima and media thickness as a risk factor for myocardial infarction and stroke in older adults. N Engl J Med 340:14-22

50. Freedman BI, Langefeld CD, Lohman KK et al (2005) Relationship between albuminuria and cardiovascular disease in type 2 diabetes. J Am Soc Nephrol 16:2156-2161

51. Bell NH, Williamson B, Hollis B, Riggs BL (2001) Effects of race on diurnal patterns of renal conservation of calcium and bone resorption in premenopausal women. Osteoporos Int $12: 43-48$

52. Dwyer JH, Dwyer KM, Scribner RA et al (1998) Dietary calcium, calcium supplementation, and blood pressure in African American adolescents. Am J Clin Nutr 68:648-655

53. Aloia J, Vaswani A, Yeh J, Flaster E (1996) Risk for osteoporosis in black women. Calcif Tissue Int 59:415-423

54. Doherty TM, Fitzpatrick LA, Shaheen A, Rajavashisth TB, Detrano RC (2004) Genetic determination of arterial calcification associated with atherosclerosis. Mayo Clin Proc 79:197210 